\title{
Preemptive pregabalin for postoperative analgesia during minimally invasive hysterectomy: a systematic review and meta-analysis of randomized controlled trials
}

\author{
Ahmed Abu-Zaid, MD ${ }^{1,2}$, Osama Alomar, MD ${ }^{1,3}$, Nora F AlNaim, MD³, Fatimah Shakir Abualsaud, MD, \\ Mohammed Ziad Jamjoom, MD³, Latifa F AINaim, MD³, Abdullah AMA Almubarki, MD, \\ Saeed Baradwan, MD ${ }^{4}$ Saud Abdullah Saud Aboudi, MD ${ }^{5}$, Faisal Khalid Idris, MD, Meshael Fodaneel, MD $^{3}$, \\ Ismail A Al-Badawi, MD, FRCSC ${ }^{1,3}$, Hany Salem, MD ${ }^{1,3}$ \\ Department of Obstetrics and Gynecology, ${ }^{1}$ College of Medicine, Alfaisal University, Riyadh, Saudi Arabia; ${ }^{2}$ College of Graduate Health Sciences, \\ University of Tennessee Health Science Center, Memphis, TN, USA; ${ }^{3}$ King Faisal Specialist Hospital and Research Center, Riyadh; ${ }^{4}$ King Faisal Specialist \\ Hospital and Research Center, Jeddah; ${ }^{5}$ College of Medicine, Prince Sattam Bin Abdulaziz University, AlKharj, Saudi Arabia
}

We aimed to perform a systematic review and meta-analysis of all randomized placebo-controlled trials (RCTs) that examined the analgesic benefits of preemptive pregabalin among patients undergoing minimally invasive hysterectomy. Five major databases were systematically screened from inception until August 29, 2021 Relevant studies were evaluated for risk of bias. Endpoints were analyzed using the random-effects model and pooled as the mean difference or risk ratio with a $95 \%$ confidence interval. Four studies with seven treatment arms met the inclusion criteria. The total sample size was 304 patients: 193 and 111 patients were allocated to the pregabalin and placebo groups, respectively. Overall, the included studies revealed a low risk of bias. The summary results revealed that the mean postoperative pain scores at rest were significantly lower in the pregabalin group than in the control group at $0,2,4,6,12$, and 24 hours. Moreover, the mean postoperative pain scores on movement/coughing were significantly lower in the pregabalin group than in the control group at 12 and 24 hours. The rate of patients who were opioid-free postoperatively was significantly higher in the pregabalin group than in the control group. There was no significant difference between the groups in terms of the mean postoperative time to first rescue analgesic and the rates of adverse events. Compared with placebo, preemptive pregabalin was largely safe, and was correlated with superior analgesic effects in terms of lower postoperative pain scores and higher opioid-sparing effects. Additional RCTs are needed to confirm these findings.

Keywords: Pregabalin; Pain; Hysterectomy; Meta-analysis

\section{Introduction}

Vaginal, laparoscopic, and robotic hysterectomies are regarded as minimally invasive procedures [1]. They are favorably associated with better quality of life, shorter hospital stay, and lower complication rates compared with abdominal hysterectomy $[2,3]$. Nonetheless, severe postoperative pain remains a major concern among patients undergoing minimally invasive hysterectomy [4-6].

Opioid treatment is the mainstay of management for acute postoperative pain following minimally invasive hysterectomy [7]. Although opioids deliver optimal pain relief, they are nonetheless correlated with a number of toxicities that
Received: 2021.11.15. Revised: 2022.01.07. Accepted: 2022.02.03. Corresponding author: Ahmed Abu-Zaid, MD

Department of Obstetrics and Gynecology, College of Graduate Health Sciences, University of Tennessee Health Science Center, 920 Madison Ave \#807, Memphis, TN 38103, USA

E-mail: aabuzaid@live.com

https://orcid.org/0000-0003-2286-2181

Articles published in Obstet Gynecol Sci are open-access, distributed under the terms of the Creative Commons Attribution Non-Commercial License (http://creativecommons. org/licenses/by-nc/3.0/) which permits unrestricted non-commercial use, distribution, and reproduction in any medium, provided the original work is properly cited.

Copyright $\odot 2022$ Korean Society of Obstetrics and Gynecology 


\section{Obstetrics \& Gynecology Science}

Vol. 65, No. 2, 2022

warrant their cessation. Examples of such toxicities include constipation, nausea, vomiting, opioid addiction, drowsiness, and hypoventilation [8]. Therefore, there is a pressing demand to investigate alternative pharmacological agents that can favorably reduce patient-reported scores for postoperative pain, opioid consumption, and adverse events of multimodal analgesia.

Pregabalin belongs to the class of gabapentinoids. Structurally, pregabalin is analogous to gamma-aminobutyric acid. Functionally, it blocks the alpha-2-delta subunit of voltagegated calcium channels that are ubiquitously expressed in the brain and spinal cord. Consequently, pregabalin blocks the release of excitatory neurotransmitters, such as norepinephrine, glutamate, and substance $P$. Thus, pregabalin eventually provides central and peripheral analgesic activities [9-11]. These anti-nociceptive properties have facilitated the use of pregabalin in the management of a wide array of acute and chronic pain conditions, including postoperative pain [12-17].

In the context of gynecology, a recent meta-analysis of randomized controlled trials (RCTs) concluded that preemptive administration of pregabalin was not clinically superior to placebo in terms of decreasing pain scores and opioid intake among patients undergoing abdominal hysterectomy [18]. A limited number of RCTs have investigated the efficacy of preemptive pregabalin on postsurgical pain after minimally invasive hysterectomy [19-22]. However, results of these RCTs have been conflicting and limited by small sample sizes and dissimilar doses. Notably, the different preemptive pregabalin doses resulted in inconsistent efficacy findings, warranting the need to conduct a meta-regression analysis to explore the moderating impact of preemptive pregabalin dose on the efficacy and safety endpoints. In addition, no meta-analysis to date has been performed to compile evidence and generate high-quality endorsements on the subject. Such an investigation is essential to inform evidence-based clinical decisions.

Hence, the objective of this investigation was to perform a systematic review and meta-analysis of all RCTs that examined the analgesic benefits of preemptive pregabalin among patients undergoing minimally invasive hysterectomy.

\section{Methods}

\section{Protocol and registration}

This investigation was carried out in compliance with the Preferred Reporting Items for Systematic Reviews and MetaAnalyses (PRISMA) [23] and the guidelines of the Cochrane Handbook for Systematic Reviews of Interventions [24]. Ethical approval was not required, as this investigation included previously published literature.

\section{Eligibility criteria}

Studies that met the following evidence-based PICOS inclusion criteria were considered appropriate: (i) patients: individuals undergoing minimally invasive hysterectomy, including vaginal, laparoscopic, and robotic approaches; (ii) intervention: preemptive pregabalin; (iii) comparator: preemptive placebo; (iv) outcomes: any of the prespecified endpoints; and (v) study design: RCTs. The exclusion criteria were as follows: (i) patients undergoing abdominal hysterectomy; (ii) interventional preemptive drugs other than pregabalin; (iii) control of preemptive drugs other than passive placebo; and (iv) study designs other than RCTs, including non-randomized studies, reviews, and conference abstracts.

\section{Information sources}

Five major electronic databases were screened from inception until August 28, 2021. These databases included PubMed, Web of Science, the Cochrane Central Register of Controlled Trials, Embase, and Scopus. Additionally, the reference lists of the included RCTs and recent reviews were manually screened for additional pertinent RCTs that could have been overlooked. There were no restrictions on patient age, language, or year of publication.

\section{Literature search}

The following query search was utilized in all five major databases: pregabalin AND hysterectomy AND (vaginal OR laparoscopic OR robotic OR minimally invasive). The literature search was independently completed by two investigators, and disagreements, if any, were rectified by consensus. Supplementary Table 1 shows the exact literature search strategy for all databases.

\section{Study selection}

All citations retrieved from the database search were im- 


\section{Obstetrics \& Gynecology Science}

Ahmed Abu-Zaid, et al. Preemptive pregabalin during MIH

ported into an Excel spreadsheet. Duplicate citations were omitted. Next, titles and/or abstracts were screened for potential eligibility and unrelated citations were removed. Subsequently, the remaining eligible citations underwent full-text examination to determine the final eligibility for inclusion in the meta-analysis. The study selection was independently completed by two investigators, and disagreements, if any, were rectified by consensus.

\section{Data collection process and items}

A data collection sheet was designed to summarize the extracted data. There were two main types of data: the first type included the baseline characteristics of the included RCTs, such as study identifier, country of publication, type of minimally invasive hysterectomy, study arms, sample sizes, age/weight/height/body mass index of patients, operation time, and details of pregabalin administration. The second type of data included the following study's endpoints: (i) the mean postoperative pain scores at rest at $0,2,4,6,12$, and 24 hours; (ii) the mean postoperative pain scores on movement/coughing at 12 and 24 hours; (iii) the rate of patients who were opioid-free postoperatively; (iv) the mean postoperative time to first analgesic rescue therapy in hours (intravenous tramadol $50 \mathrm{mg}$ or intramuscular diclofenac sodium $1 \mathrm{mg} / \mathrm{kg}$ ); (v) the rates of patients with nausea only, nausea plus vomiting, vomiting only, mouth dryness, and respiratory depression; (vi) mean Ramsay sedation score; and (vii) rate of patients with $\geq 3$ Ramsay sedation score within 0-1 hours postoperatively [25]. The postoperative pain scores were assessed in accordance with the 10-cm visual analog scale (VAS), in which "0" corresponded to no pain at all and "10" corresponded to the worst possible pain imaginable. Postoperative opioid consumption included either pethidine (50-100 mg) or tramadol (50 mg), as reported in the relevant RCTs. Ramsay sedation score is a well-established metric used to characterize a person's level of sedation (sleepiness). It has six possible scores, as follows: patient is anxious and agitated or restless, or both (score 1); patient is cooperative, oriented, and tranquil (score 2); patient responds to commands only (score 3); patient exhibits brisk response to light glabellar tap or loud auditory stimulus (score 4); patient exhibits a sluggish response to light glabellar tap or loud auditory stimulus (score 5); and patient exhibits no response (score 6) [25]. Data were collected by six investigators (in groups of two) independently, and disagreements, if any, were rectified by consensus among the groups.

\section{Risk of bias evaluation of the included studies}

The Cochrane risk of bias tool was used to assess the quality of the included studies [26]. Seven key domains were evaluated: (i) random sequence generation, (ii) allocation concealment, (iii) performance bias, (iv) detection bias, (v) attrition bias, (vi) reporting bias, and (vii) other potential sources of bias. Each domain was scored as high, low, or unclear. The risk of bias evaluation of the included studies was completed independently by two investigators, and disagreements, if any, were rectified by consensus.

\section{Summary measures and data analysis}

Continuous data were analyzed using the inverse variance method and summarized as mean difference (MD) with 95\% confidence interval $(\mathrm{CI})$. Dichotomous data were analyzed using the Mantel-Haenszel method and summarized as risk ratio (RR) with $95 \% \mathrm{Cl}$. Considering that the effects estimated across the different RCTs were not identical, the random-effects model was used [24]. Between-study heterogeneity was defined based on two conditions: (i) the $P$-value of Cochran's Q test $(P<0.1)$, and (ii) the I-square $\left(I^{2}\right)$ test $\left(I^{2}>50 \%\right)$ [27]. The Review Manager Software, version 5.4.0 for Windows (Cochrane, London, UK), was used for statistical analysis of the forest plots. Leave-one-out sensitivity analysis was performed to examine the robustness of the overall findings by eliminating one study at each analysis and recalculating the overall effect sizes of the remaining studies [24]. Meta-regression analysis was performed to explore the moderator effect of preemptive pregabalin dose on all outcomes, and the results were depicted in bubble plots. Publication bias was assessed virtually through funnel plots and quantitatively using Egger's regression test [28]. The STATA software for Windows (version 17.0; StataCorp LLC, College Station, TX, USA) was used for performing leave-one-out sensitivity analysis, metaregression analysis (bubble plots), and publication bias (funnel plots). One study [22] reported medians and range values (minimum and maximum), and the means and standard deviations were computed as outlined previously by Wan et al. [29]. Two studies $[19,21]$ included more than one dose of pregabalin, and we treated each dose as a standalone RCT (versus control) in this meta-analysis, consistent with earlier reports $[30,31]$. 


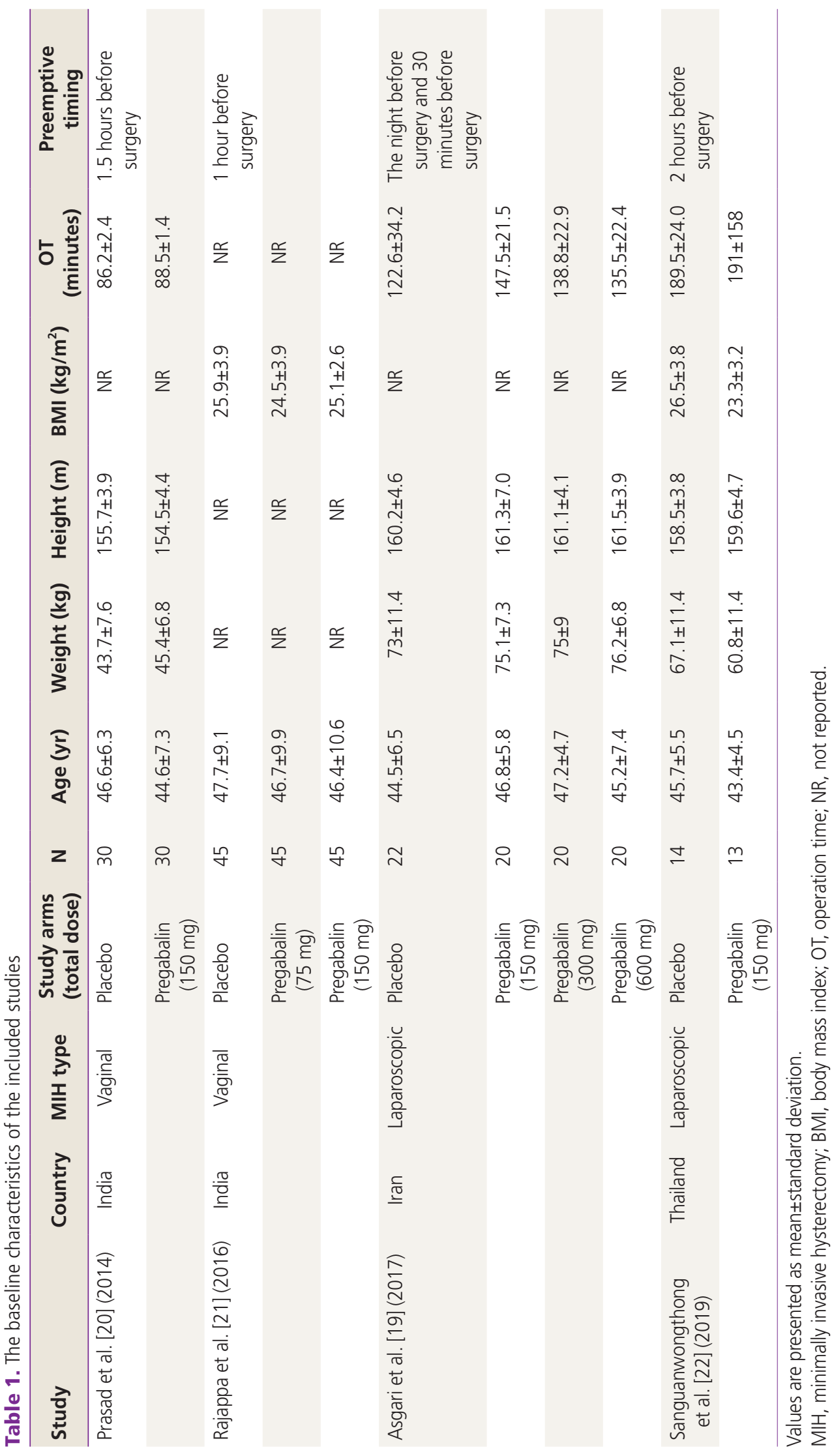




\section{Obstetrics \& Gynecology Science}

Ahmed Abu-Zaid, et al. Preemptive pregabalin during $\mathrm{MIH}$

\section{Results}

\section{Literature search}

Supplementary Fig. 1 summarizes the PRISMA flowchart used for the literature search. Overall, a literature search identified 102 suitable studies. After eliminating duplicate studies $(n=44)$, the remaining studies $(n=58)$ underwent screening of titles and abstracts, and 52 studies were excluded. Six studies underwent full-text reading, and only four unique studies were considered eligible for inclusion in the meta-analysis [19-22]. Table 1 summarizes the baseline characteristics of the included studies. Overall, this meta-analysis included four studies with seven treatment arms based on pregabalin dose. The total sample size was 304 patients. There were 193 and 111 patients allocated to the pregabalin and control groups, respectively. All studies were published between 2014 and 2019 and took place in India $(n=2)$, Iran $(n=1)$, and Thailand $(n=1)$. Laparoscopic and vaginal approaches to hysterectomy were performed in two studies each. The total dose of preemptive pregabalin ranged from 75 to $600 \mathrm{mg}$.

\section{Risk of bias of the included studies}

Supplementary Fig. 2 summarizes the risk of bias in the included studies. Overall, the included studies revealed a low risk of bias. One study [21] did not provide adequate information about the detection bias, and this corresponding domain was scored as unclear risk.

\section{Meta-analysis of the mean postoperative pain scores at rest at $\mathbf{0 ,}, 2$, and $\mathbf{4}$ hours}

The summary results revealed that the mean postoperative pain scores at rest were significantly lower in the pregabalin group than in the control group at 0 hours $(n=4 R C T s ; M D$, $-2 ; 95 \% \mathrm{Cl},-3.38$ to $-0.62 ; P=0.004), 2$ hours ( $n=4$ RCTs; MD, $-2.5 ; 95 \% \mathrm{Cl},-3.51$ to $-1.49 ; P<0.001)$, and 4 hours $(n=4$ RCTs; MD, $-1.17 ; 95 \% \mathrm{Cl},-1.84$ to $-0.49 ; P<0.001)$. The pooled results were heterogeneous (Fig. 1).

\section{Meta-analysis of the mean postoperative pain scores at rest at 6,12 , and 24 hours}

The summary results revealed that the mean postoperative pain scores at rest were significantly lower in the pregabalin

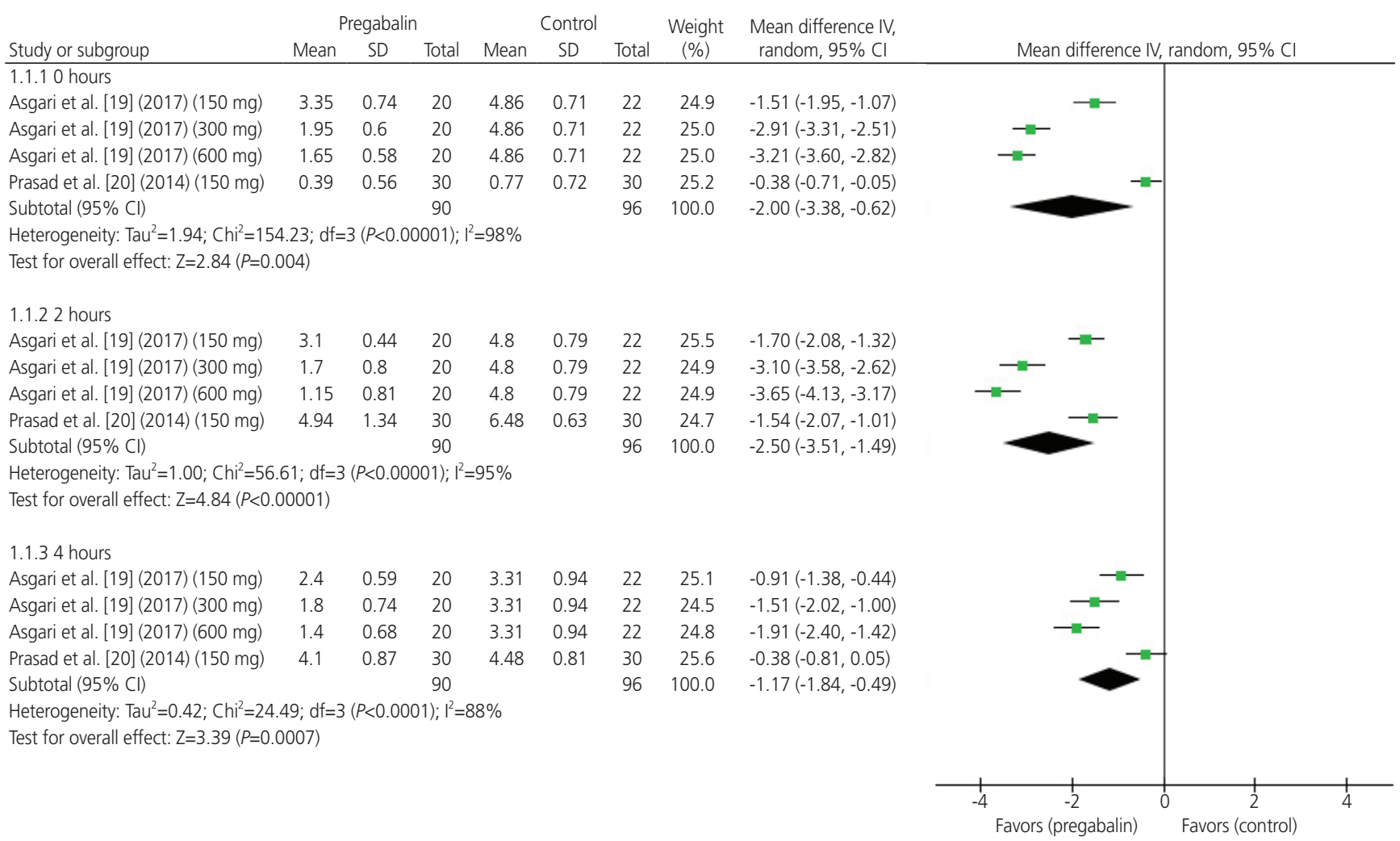

Fig. 1. Meta-analysis of the mean postoperative pain scores at rest at 0,2 , and 4 hours. SD, standard deviation; $\mathrm{Cl}$, confidence interval. 


\section{Obstetrics \& Gynecology Science}

Vol. 65, No. 2, 2022

group than in the control group at 6 hours ( $n=7$ RCTs; MD, $-0.97 ; 95 \% \mathrm{Cl},-1.39$ to $-0.55 ; P<0.001), 12$ hours $(n=7$ RCTs; MD, $-1.37 ; 95 \% \mathrm{Cl},-2.32$ to $-0.42 ; P=0.005)$, and 24 hours ( $n=7$ RCTs; MD, $-1.23 ; 95 \% \mathrm{Cl},-2.01$ to -0.45 ; $P=0.002$ ). The pooled results were heterogeneous (Fig. 2).

\section{Meta-analysis of the mean postoperative pain scores on moving/coughing at 12 and 24 hours}

The summary results revealed that the mean postoperative pain scores on moving/coughing were significantly lower in the pregabalin group than in the control group at 12 hours ( $n=5$ RCTs; MD, $-1.08 ; 95 \% \mathrm{Cl}_{1}-2.1$ to $-0.06 ; P=0.04$ ) and 24 hours ( $n=5$ RCTs; MD, $-1.21 ; 95 \% \mathrm{Cl},-2.28$ to 0.15 ; $P=0.03$ ). The pooled results were heterogeneous (Fig. 3).

\section{Meta-analysis of the rate of patients who were opioid-free postoperatively}

The rate of patients who were opioid-free postoperatively was significantly higher in the pregabalin group than in the control group ( $n=5$ RCTs; RR, $7.13 ; 95 \% \mathrm{Cl}, 3.72$ to 13.67; $P<0.001)$. The pooled results were homogeneous (Fig. 4). Sanguanwongthong et al. [22] showed that the mean postoperative opioid (fentanyl) intake was significantly lower in the pregabalin group than in the control group (133.92 \pm 77.94 vs. $337.63 \pm 178.47 \mathrm{mg}, P=0.001)$.

\section{Meta-analysis of the mean postoperative time to first rescue analgesic}

The mean postoperative time to first rescue analgesic did not differ significantly between the two groups ( $n=3 R C T s ; M D$, $6.65 ; 95 \% \mathrm{Cl},-1.04$ to $14.35 ; P=0.09$ ). The pooled results were heterogeneous (Fig. 5).

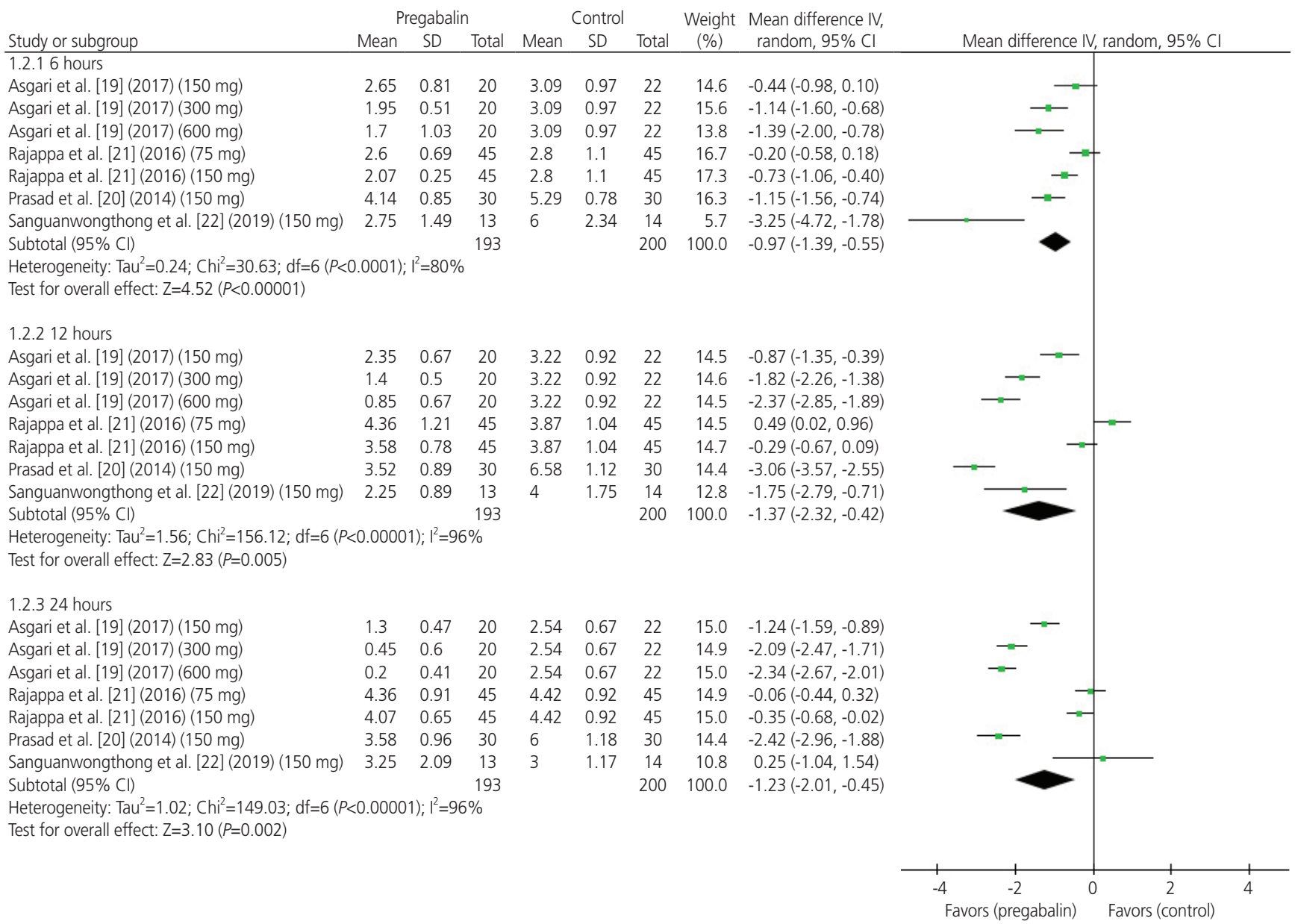

Fig. 2. Meta-analysis of the mean postoperative pain scores at rest at 6, 12, and 24 hours. SD, standard deviation; $\mathrm{Cl}$, confidence interval. 


\section{Obstetrics \& Gynecology Science}

Ahmed Abu-Zaid, et al. Preemptive pregabalin during MIH

\section{Meta-analysis of the rates of nausea only, nausea plus vomiting, vomiting only, mouth dryness, and respiratory depression}

The rates of nausea only ( $n=6$ RCTs; $R R, 1.09 ; 95 \% \mathrm{Cl}, 0.98$ to $1.21 ; P=0.1$ ), nausea plus vomiting ( $n=4 R C T s ; R R, 0.86$;
$95 \% \mathrm{Cl}, 0.44$ to $1.66 ; P=0.65$ ), vomiting only ( $\mathrm{n}=2 \mathrm{RCTs}$; $R$, $1.13 ; 95 \% \mathrm{Cl}, 0.73$ to $1.75 ; P=0.59)$, and mouth dryness ( $n=1$ $\mathrm{RCT} ; \mathrm{RR}, 2 ; 95 \% \mathrm{Cl}, 0.19$ to $20.9 ; P=0.56)$ did not differ significantly between the two groups. The pooled results were homogeneous (Supplementary Fig. 3). One RCT by Prasad et

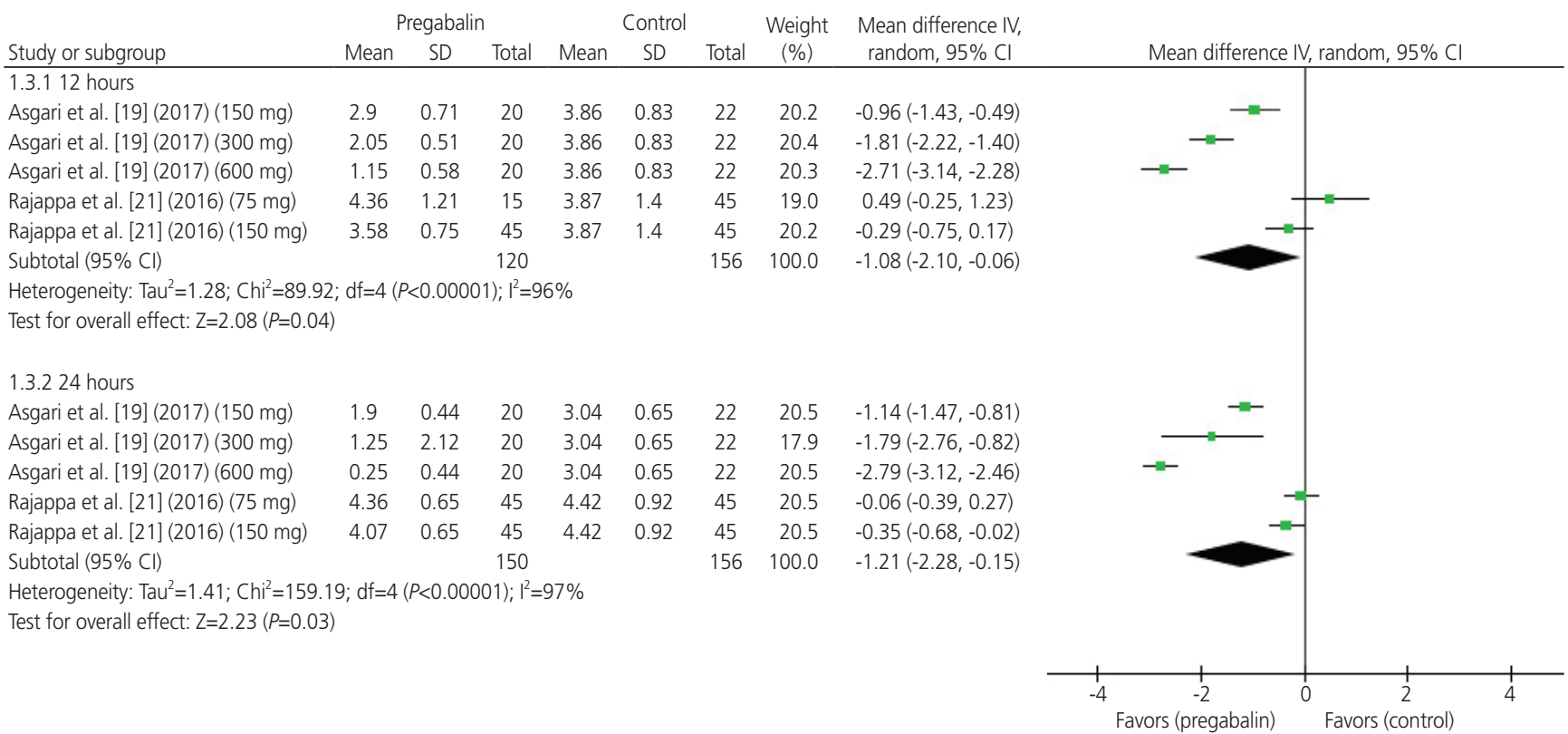

Fig. 3. Meta-analysis of the mean postoperative pain scores on moving/coughing at 12 and 24 hours. SD, standard deviation; $\mathrm{Cl}$, confidence interval.

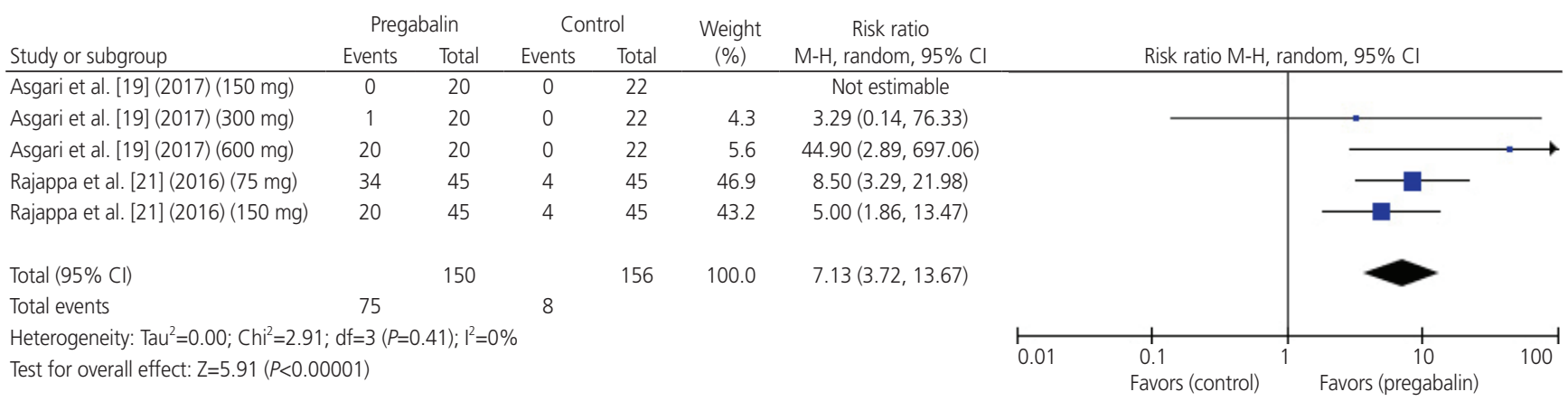

Fig. 4. Meta-analysis of the rate of patients who were opioid-free postoperatively. $\mathrm{Cl}$, confidence interval.

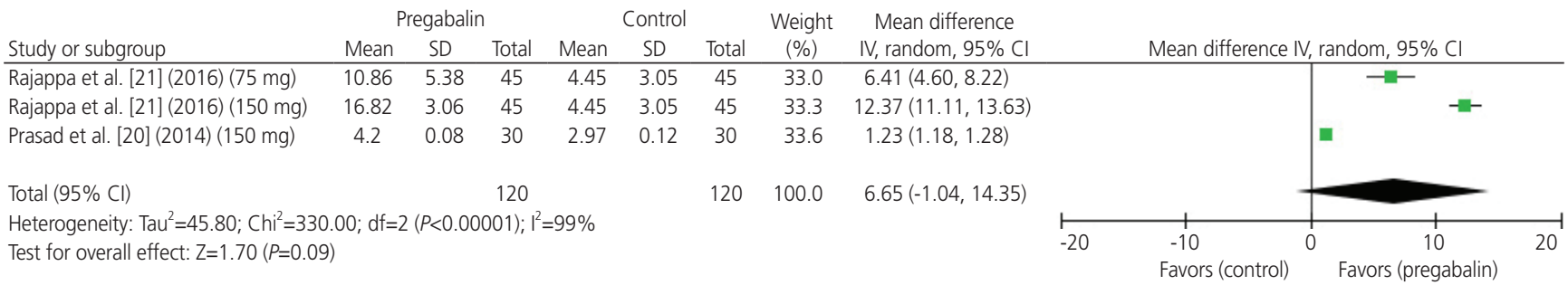

Fig. 5. Meta-analysis of the mean postoperative time to first analgesic rescue. SD, standard deviation; $\mathrm{Cl}$, confidence interval. 


\section{Obstetrics \& Gynecology Science}

Vol. 65 , No. 2, 2022

al. [20] reported respiratory depression as a potential side effect, and no single event was documented in either group.

\section{Meta-analysis of the mean Ramsay sedation score and the rate of patients with $\geq 3$ Ramsay sedation score within 0-1 hours postoperatively}

The mean postoperative Ramsay sedation score did not significantly differ between the two groups ( $n=4 R C T s ; M D$, $1.04 ; 95 \% \mathrm{Cl},-0.57$ to $2.64 ; P=0.21$ ). The pooled analysis was heterogeneous (Supplementary Fig. 4A). However, the rate of patients with $\geq 3$ Ramsay sedation score within 0-1 hours postoperatively was significantly higher in the pregabalin group than in the control group ( $n=3$ RCTs; RR, 16.23; $95 \% \mathrm{Cl}, 4.61$ to $57.21 ; P<0.001)$. The pooled analysis was homogeneous (Supplementary Fig. 4B).

\section{Leave-one-out sensitivity analysis}

The results of leave-one-out sensitivity analysis demonstrated robustness for all outcomes, except for mean postoperative pain scores at rest at 0 hours, mean postoperative pain scores on motion/coughing at 12 hours, mean postoperative pain scores on motion/coughing at 24 hours, and mean postoperative time to first analgesic rescue (Supplementary Figs. 5-9). For the non-robust outcomes, omission of some individual RCTs exhibited a significant impact on the overall summary effect sizes of the rest of the RCTs.

\section{Meta-regression analysis}

Meta-regression based on the dose of preemptive pregabalin as a moderator showed a significant impact on the mean postoperative pain scores at rest and on motion/coughing (Supplemental Figs. 10, 11), and an inversely proportional relationship was observed in which higher preemptive pregabalin doses correlated with a greater decrease in mean postoperative pain scores. Moreover, meta-regression based on the dose of preemptive pregabalin as a moderator showed a significant impact on mean postoperative Ramsay sedation score (Supplementary Fig. 12A), and a directly proportional relationship was observed in which higher preemptive pregabalin doses correlated with a higher mean postoperative Ramsay sedation score. However, meta-regression based on the dose of preemptive pregabalin as a moderator did not show a significant impact on the other endpoints (Supplementary Figs. 12B, 13, 14).

\section{Publication bias}

Funnel plots and Egger's test results for all outcomes are depicted in Supplementary Figs. 15-19. Visual inspection of the funnel plots revealed no significant symmetry, except for the mean postoperative time to the first analgesic rescue and the mean postoperative Ramsay sedation score.

\section{Discussion}

This systematic review and meta-analysis examined the analgesic utility of preemptive pregabalin among patients undergoing minimally invasive hysterectomy. Four studies with seven different treatment arms were analyzed (193 and 111 patients were assigned to the pregabalin and control groups, respectively). The included studies revealed an overall low risk of bias. Our results revealed that preemptive pregabalin correlated with significantly reduced postoperative pain scores at rest and on moving/coughing. Additionally, preemptive pregabalin was associated with a higher rate of postoperative opioid-free patients. Sedation was a significant adverse event in the preemptive pregabalin group compared with the control group. There was no significant difference between the groups regarding the rates of other adverse events, and these adverse events tended to increase with higher doses.

Preemptive analgesia has the advantage of preventing the onset of nociceptive stimuli and decreasing the degree of postoperative pain [32]. Pregabalin has anxiolytic, anticonvulsant, and most importantly, analgesic properties $[10,12]$. High-quality evidence from meta-analyses of RCTs demonstrated the analgesic efficacy of preemptive pregabalin in decreasing postsurgical pain score and opioid consumption among patients undergoing surgery for breast cancer [14], total joint arthroplasty [15], thoracotomy [16], and laparoscopic cholecystectomy [33]. The present meta-analysis echoes the aforementioned studies and corroborates the analgesic utility of preemptive pregabalin in controlling postoperative pain and minimizing opioid intake among patients undergoing minimally invasive hysterectomy. Our findings are in opposition to a meta-analysis that demonstrated no analgesic advantages of preemptive pregabalin among patients undergoing abdominal hysterectomy [18]. One potential reason for this observed conflict could be ascribed to the fact that minimally invasive hysterectomy is associated with less trauma to the body than abdominal hysterectomy, in terms 


\section{Obstetrics \& Gynecology Science}

Ahmed Abu-Zaid, et al. Preemptive pregabalin during MIH

of less incisional injury, inflammation, and neuropathic insult [34]. Hence, pain perception is anticipated to be intrinsically lower in minimally invasive hysterectomy than in abdominal hysterectomy. Therefore, preemptive pregabalin is projected to exhibit more analgesic benefits among patients undergoing minimally invasive hysterectomy than those undergoing abdominal hysterectomy.

Poor control of postoperative pain is associated with a number of unfavorable outcomes. Such outcomes include increased chronic opioid addiction, delayed mobilization, poor quality of life, prolonged hospitalization, delayed return to normal activities, higher hazard of postoperative complications, and increased patient care expenses [5]. The minimum clinically important difference for alleviation of acute postoperative pain is a reduction of at least 1 point out of the 10-point VAS [35]. Our meta-analysis showed that the reductions in postsurgical pain scores at rest and on motion/coughing were statistically significant and clinically important. Higher analgesic benefits, in terms of postoperative pain scores and opioid-sparing effects, were observed at higher doses, suggesting a dose-response relationship. This phenomenon was previously reported in a network metaanalysis of 79 RCTs [36].

Sedation was a significant adverse event of preemptive pregabalin in our meta-analysis, which is consistent with previous literature [37]. Moreover, our meta-analysis revealed no significant toxicity of pregabalin compared with the control regarding the rates of nausea only, vomiting only, nausea plus vomiting, mouth dryness, and respiratory depression. However, the abovementioned toxicities tended to increase with higher doses, suggesting a potential dose-dependent correlation [36]. Thus, the analgesic benefits of preemptive pregabalin should be carefully balanced against anticipated toxicities.

Gabapentin is a gabapentinoid drug that is closely related to pregabalin $[9,10]$. A recent meta-analysis of $\operatorname{RCTs}(n=14)$ demonstrated the analgesic efficacy of preemptive gabapentin among patients undergoing hysterectomy [38]. Overall, 12 and two RCTs involved patients undergoing abdominal and minimally invasive hysterectomy, respectively. The study concluded that administration of preemptive gabapentin correlated with reduced postoperative pain scores, better opioid-sparing sparring effects, higher patient satisfaction, and lower incidence rates of nausea and vomiting. Nonetheless, the clinical utility and safety of preemptive gabapentin have yet to be comprehensively evaluated among patients undergoing minimally invasive hysterectomy.

Prospective investigations may include conducting RCTs comparing pregabalin with active comparators (for example, gabapentin or para-cervical block with bupivacaine) [39] among patients undergoing minimally invasive hysterectomy. The optimal dose of preemptive pregabalin has yet to be determined. Thus, additional studies may involve conducting a dose-response analysis to identify the preemptive pregabalin dose that will achieve the maximum analgesic effect and minimum toxicity. Furthermore, it will be of interest to identify the subset of patients who are likely to gain maximum benefit from preemptive pregabalin during minimally invasive hysterectomy. Lastly, future investigations may include examining whether pregabalin administered preemptively and postoperatively will correlate with better analgesia compared with preemptive administration only.

In this meta-analysis, the data on publication bias should be interpreted with extreme caution for three main reasons. First, funnel plots were assessed subjectively; hence, the results were liable to underestimation or overestimation of publication bias [40]. Second, it remains uncertain whether funnel plots can properly identify publication bias [41]. Third, when the total number of included studies is less than 10 RCTs per outcome, the visual inspection of funnel plots and their quantitative methods (for example, Egger's regression test) become unreliable $[28,42]$.

Our investigation has a number of strengths that should be acknowledged. We performed the first meta-analysis to scrutinize the analgesic efficacy and safety of preemptive pregabalin among patients undergoing minimally invasive hysterectomy. We conducted PRISMA-compliant research, screened five major databases, and applied strict eligibility criteria. As opposed to other study designs, we considered only RCTs to facilitate the generation of high-quality evidence. Quality assessment of the eligible RCTs exhibited an overall low risk of bias, highlighting the validity of the conclusions. Additionally, we reported several endpoints and dissected the pregabalin doses. Lastly, we enriched our investigation with leave-oneout sensitivity analysis, meta-regression analysis, and funnel plot assessment for publication bias for all outcomes.

Nonetheless, our investigation also has some limitations. These limitations include the relatively small number of eligible RCTs and their corresponding small sample sizes. Moreover, in most of the comparisons, data were analyzed from 


\section{Obstetrics \& Gynecology Science}

Vol. 65, No. 2, 2022

only two studies. Both limitations may have played key roles in the observed between-study heterogeneity of the reported outcomes. Additional limitations include the different patient demographics (for example, type of minimally invasive hysterectomy) and pregabalin administration (for example, the variance of dose and schedule), which could have impacted the reported endpoints and culminated in higher betweenstudy heterogeneity. Moreover, the included RCTs failed to document other relevant endpoints, such as hospitalization length, chronic pain control at three months, patient satisfaction, and rapidity of return to normal activities.

\section{Conclusion}

Among patients undergoing minimally invasive hysterectomy, preemptive pregabalin was safe and correlated with superior analgesic effects in terms of lower postoperative pain scores and higher opioid-sparing effects compared with placebo. Owing to the unavoidable limitations of this meta-analysis, the conclusions should be interpreted with caution, and additional RCTs are needed to validate these findings.

\section{Conflict of interest}

The authors declare that they have no conflicts of interest.

\section{Ethical approval}

This study does not require approval of the Institutional Review Board because no patient data is contained in this article. The study was performed in accordance with the principles of the Declaration of Helsinki.

\section{Patient consent}

Written informed consent and the use of images from patients are not required for the publication.

\section{Funding information}

None.

\section{Supplementary material}

Supplementary Table 1 and Figs. 1-19 associated with this article can be found online at https://doi.org/10.5468/ ogs.21345.

\section{References}

1. Committee Opinion No 701: choosing the route of hysterectomy for benign disease. Obstet Gynecol 2017;129:e155-9.

2. Johnson N, Barlow D, Lethaby A, Tavender E, Curr L, Garry R. Methods of hysterectomy: systematic review and meta-analysis of randomised controlled trials. BMJ 2005;330:1478.

3. O'Neill M, Moran PS, Teljeur C, O'Sullivan OE, O'Reilly BA, Hewitt M, et al. Robot-assisted hysterectomy compared to open and laparoscopic approaches: systematic review and meta-analysis. Arch Gynecol Obstet 2013;287:907-18.

4. Tulandi T, Krishnamurthy $S$, Mansour F, Suarthana E, AlMalki G, Ballesteros LER, et al. A triple-blind randomized trial of preemptive use of gabapentin before laparoscopic hysterectomy for benign gynaecologic conditions. J Obstet Gynaecol Can 2019;41:1282-8.

5. Benton A, Harkins G, Stetter C, Kunselman A, Deimling T, Riley K. Administration of preoperative gabapentin to patients undergoing laparoscopy: a double-blinded, placebo-controlled randomized trial. J Gynecol Surg 2020;36:173-8.

6. As-Sanie S, Till SR, Mowers EL, Lim CS, Skinner BD, Fritsch L, et al. Opioid prescribing patterns, patient use, and postoperative pain after hysterectomy for benign indications. Obstet Gynecol 2017;130:1261-8.

7. Weston E, Raker C, Huang D, Parker A, Cohen M, Robison $\mathrm{K}$, et al. Opioid use after minimally invasive hysterectomy in gynecologic oncology patients. Gynecol Oncol 2019;155:119-25.

8. Benyamin R, Trescot AM, Datta S, Buenaventura R, Ad- 


\section{Obstetrics \& Gynecology Science}

Ahmed Abu-Zaid, et al. Preemptive pregabalin during MIH

laka R, Sehgal N, et al. Opioid complications and side effects. Pain Physician 2008;11(2 Suppl):S105-20.

9. Sills GJ. The mechanisms of action of gabapentin and pregabalin. Curr Opin Pharmacol 2006;6:108-13.

10. Bockbrader HN, Wesche D, Miller R, Chapel S, Janiczek $\mathrm{N}$, Burger P. A comparison of the pharmacokinetics and pharmacodynamics of pregabalin and gabapentin. Clin Pharmacokinet 2010;49:661-9.

11. Calandre EP, Rico-Villademoros F, Slim M. Alpha2delta ligands, gabapentin, pregabalin and mirogabalin: a review of their clinical pharmacology and therapeutic use. Expert Rev Neurother 2016;16:1263-77.

12. Dauri M, Faria S, Gatti A, Celidonio L, Carpenedo R, Sabato AF. Gabapentin and pregabalin for the acute postoperative pain management. A systematic-narrative review of the recent clinical evidences. Curr Drug Targets 2009;10:716-33.

13. Clarke H, Bonin RP, Orser BA, Englesakis M, Wijeysundera DN, Katz J. The prevention of chronic postsurgical pain using gabapentin and pregabalin: a combined systematic review and meta-analysis. Anesth Analg 2012;115:428-42.

14. Chang CC, Yen WT, Lin YT, Wang LK, Hung KC, Wu ZF, et al. Perioperative pregabalin for preventive analgesia in breast cancer surgery: a meta-analysis of randomized controlled trials. Clin J Pain 2020;36:968-77.

15. Hannon $C P$, Fillingham YA, Browne JA, Schemitsch EH, Mullen K, Casambre F, et al. The efficacy and safety of gabapentinoids in total joint arthroplasty: systematic review and direct meta-analysis. J Arthroplasty 2020;35:2730-8.e6.

16. Yu Y, Liu N, Zeng Q, Duan J, Bao Q, Lei M, et al. The efficacy of pregabalin for the management of acute and chronic postoperative pain in thoracotomy: a metaanalysis with trial sequential analysis of randomizedcontrolled trials. J Pain Res 2018;12:159-70.

17. Wang SL, Wang H, Nie HY, Bu G, Shen XD, Wang H. The efficacy of pregabalin for acute pain control in herpetic neuralgia patients: a meta-analysis. Medicine (Baltimore) 2017;96:e9167.

18. Ni J, Jiang J, Mao S, Sun RF. Pregabalin does not decrease acute pain or postoperative nausea and vomiting after hysterectomy: a meta-analysis. J Int Med Res 2020;48: 300060520954720.

19. Asgari Z, Rouholamin S, Nataj M, Sepidarkish M, Hos- seini $R$, Razavi M. Dose ranging effects of pregabalin on pain in patients undergoing laparoscopic hysterectomy: a randomized, double blinded, placebo controlled, clinical trial. J Clin Anesth 2017;38:13-7.

20. Prasad A, Bhattacharyya S, Biswas A, Saha M, Mondal S, Saha D. A comparative study of pre-operative oral clonidine and pregabalin on post-operative analgesia after spinal anesthesia. Anesth Essays Res 2014;8:41-7.

21. Rajappa GC, Vig S, Bevanaguddaiah Y, Anadaswamy TC. Efficacy of pregabalin as premedication for post-operative analgesia in vaginal hysterectomy. Anesth Pain Med 2016;6:e34591.

22. Sanguanwongthong K, Imruetaicharoenchok A, Phaloprakarn C, Vitayaburananont P. A randomized, doubleblind, placebo-controlled trial of pre-emptive pregabalin for postoperative pain after laparoscopic hysterectomy in benign gynecologic diseases. J Med Assoc Thai 2019;102:39.

23. Moher D, Liberati A, Tetzlaff J, Altman DG; PRISMA Group. Preferred reporting items for systematic reviews and meta-analyses: the PRISMA statement. PLoS Med 2009;6:e1000097.

24. Higgins J, Thomas J, Chandler J, Cumpston M, Li T, Page $M$, et al. Cochrane handbook for systematic reviews of interventions version 5.1.0 [Internet]. London (UK): The Cochrane Collaboration; c2021 [cited 2011 Sep 4]. Available from: www.handbook.cochrane.org.

25. Ramsay MA, Savege TM, Simpson BR, Goodwin R. Controlled sedation with alphaxalone-alphadolone. $\mathrm{Br}$ Med J 1974;2:656-9.

26. Higgins JP, Altman DG, Gøtzsche PC, Jüni P, Moher D, Oxman $A D$, et al. The Cochrane Collaboration's tool for assessing risk of bias in randomised trials. BMJ 2011;343: d5928.

27. Higgins JP, Thompson SG, Deeks JJ, Altman DG. Measuring inconsistency in meta-analyses. BMJ 2003;327:557-60.

28. Egger M, Davey Smith G, Schneider M, Minder C. Bias in meta-analysis detected by a simple, graphical test. BMJ 1997;315:629-34.

29. Wan X, Wang W, Liu J, Tong T. Estimating the sample mean and standard deviation from the sample size, median, range and/or interquartile range. BMC Med Res Methodol 2014;14:135.

30. Kutbi EH, Sohouli MH, Fatahi S, Lari A, Shidfar F, Aljhdali $M M$, et al. The beneficial effects of cinnamon among 


\section{Obstetrics \& Gynecology Science}

Vol. 65, No. 2, 2022

patients with metabolic diseases: a systematic review and dose-response meta-analysis of randomized-controlled trials. Crit Rev Food Sci Nutr 2021 Mar 19 [Epub]. https://doi.org/10.1080/10408398.2021.1896473.

31. Abu-Zaid A, Altowairqi AK, Dissanayaka T, Oganesyan A, Bhagavathul AS, Alhabeeb $H$, et al. A systematic review and dose-response meta-analysis on the efficacy of dapagliflozin in patients with type 1 diabetes mellitus. Pharmacol Res 2021;165:105456.

32. Gottschalk A, Smith DS. New concepts in acute pain therapy: preemptive analgesia. Am Fam Physician 2001;63:1979-84.

33. Zhang D, You G, Yao X. Influence of pregabalin on postoperative pain after laparoscopic cholecystectomy: a meta-analysis of randomised controlled trials. J Minim Access Surg 2019;16:99-105.

34. Blanton E, Lamvu G, Patanwala I, Barron KI, Witzeman $\mathrm{K}$, Tu FF, et al. Non-opioid pain management in benign minimally invasive hysterectomy: a systematic review. Am J Obstet Gynecol 2017;216:557-67.

35. Myles PS, Myles DB, Galagher W, Boyd D, Chew C, MacDonald $\mathrm{N}$, et al. Measuring acute postoperative pain using the visual analog scale: the minimal clinically important difference and patient acceptable symptom state. Br J Anaesth 2017;118:424-9.
36. Hu J, Huang D, Li M, Wu C, Zhang J. Effects of a single dose of preoperative pregabalin and gabapentin for acute postoperative pain: a network meta-analysis of randomized controlled trials. J Pain Res 2018;11:2633-43.

37. White PF, Tufanogullari B, Taylor J, Klein K. The effect of pregabalin on preoperative anxiety and sedation levels: a dose-ranging study. Anesth Analg 2009;108:1140-5.

38. Li XD, Han C, Yu WL. Is gabapentin effective and safe in open hysterectomy? A PRISMA compliant metaanalysis of randomized controlled trials. J Clin Anesth 2017; 41:76-83.

39. Noor N, Roy KK, Zangmo R, Das A, Rai R, Kumari A, et al. Role of para-cervical block in reducing immediate postoperative pain after total laparoscopic hysterectomy: a prospective randomized placebo-controlled trial. Obstet Gynecol Sci 2021;64:122-9.

40. Terrin N, Schmid CH, Lau J. In an empirical evaluation of the funnel plot, researchers could not visually identify publication bias. J Clin Epidemiol 2005;58:894-901.

41. Lau J, loannidis JP, Terrin N, Schmid CH, Olkin I. The case of the misleading funnel plot. BMJ 2006;333:597-600.

42. Sterne JA, Gavaghan D, Egger M. Publication and related bias in meta-analysis: power of statistical tests and prevalence in the literature. J Clin Epidemiol 2000;53:111929. 